\title{
STesearch Square

\section{A health care record review of early mobility activities after fragility hip fracture: Utilizing the French systematic method to inform future interventions}

\section{Lynn Haslam-Larmer ( $D$ 16lhl@queensu.ca )}

Ontario Shores Centre for Mental Health Sciences https://orcid.org/0000-0003-2554-1981

Kevin Woo

Queen's University

Mohammad Auais

Queen's University

Catherine Donnelly

Queen's University

Vincent DePaul

Queen's University

\section{Research}

Keywords: hip fracture, fragility, mobility, practice gap

Posted Date: March 9th, 2020

DOI: https://doi.org/10.21203/rs.3.rs-16282/v1

License: (c) (1) This work is licensed under a Creative Commons Attribution 4.0 International License. Read Full License

Version of Record: A version of this preprint was published at International Journal of Orthopaedic and Trauma Nursing on July 1 st, 2021. See the published version at https://doi.org/10.1016/j.ijotn.2021.100846. 


\section{Abstract}

Background A fragility hip fracture is a serious injury in older adults. Following a fragility fracture, a large percentage of patients are unable to regain their pre-fracture level of mobility. There are several international guidelines recommending early mobility after surgery. We do not know the utilization of these early mobility recommendations by health care providers within our institution. An evidence to practice gap occurs when there is a failure to implement best practices. Utilization of a systematic method allows for a strategic approach to assessment of an evidence to practice gap. A recent publication of quality standards in Ontario provides an opportunity for a local needs assessment of potential evidence to practice gaps.

Objective To identify if there is an evidence to practice gap in health care provider implementation of recommendations for early mobility after fragility hip fracture surgery.

Methods A retrospective chart review was performed to document the rates of early mobility activities during the first five days after hip fracture surgery at a large tertiary centre in Toronto, Ontario. Patients with cognitive impairment were included.

Results Early mobility activities in this older adult population are initiated in the first five days after surgery to varying degrees. Between $11 \%-50 \%$ of patients are not participating in early mobility activities, thereby not meeting recommendations. Those with low pre-fracture function and cognitive impairment have lower rates of participation when compared to those with a high pre-fracture function and no cognitive impairment.

Conclusions The chart review has identified a paucity of contextual information which may influence health care providers' behaviours related to early mobility. The chart audit is limited in its ability to assess the systems issues, which may have an influence on the health care provider behaviour. Considering the lack of information in these areas, we have identified that further work is required to explore factors which may be having an impact on the health care provider's ability to engage the patients in early mobility activities.

\section{Contributions To The Literature}

- Conducting a needs assessment through a chart review can provide insight to local contextual factors which influence clinical behaviours

- By following the French systematic method, specifically Step 1, we identify potential evidence-topractice gaps and clinical behaviours that may be a target for future change

- Early mobility guidelines following fragility hip fracture were first published over a decade ago, but we do not know how well they are implemented by clinicians

- We found that although physiotherapy assesses patients recovering from fragility fracture, the participation in early mobility activities is limited, and recommendations are not routinely 
implemented

\section{Introduction}

Every year, more than 32,500 Canadians experience a fragility hip fracture, of whom 11,000 reside in Ontario (1). Fragility hip fractures are fractures of the proximal femur caused by low-energy trauma, such as falls from a standing height (2). Following a fragility hip fracture, up to $60 \%$ of patients are unable to regain their pre-fracture level of mobility within 6 months $(3,4)$, and subsequent institutionalization occurs in up to $20 \%$ of patients (5). Literature has demonstrated that between $31 \%$ and $65 \%$ of patients with a fragility hip fracture experience a cognitive impairment, further complicating rehabilitation outcomes (6). In fact, those with cognitive impairment are known to experience higher mortality rates and are more likely to be placed into long-term care (7-9) than those without cognitive impairment.

Upon review of evidence in all stages of management of patients with hip fracture, the National Institute of Health and Care Evidence (NICE) guideline (2017) identifies early mobilization as an important element to decrease overall length of hospital stay, aid in re-establishing a patients' functional status, and return them to their pre-fracture environment (10). When mobilization is started within the first 48 hours after surgery, there are statistically and clinically significant increases in independence to transfer at day 7 , and a doubling in the distance walked (11). In 2017, Health Quality Ontario (HQO), the provincial governmentdesignated lead on health care quality, released the Hip Fracture Quality Standards, a set of recommendations focusing on high priority areas for improvement in care, one of which is early mobilization (2). A key to achieving optimal outcomes in the older adult population with fragility fracture is the adoption and utilization of best practice recommendations for early mobility after surgery $(10,12$, 13). However, the extent that health care providers have implemented the recommendations in their day to day practice remains mostly unknown.

An evidence-to-practice gap occurs with a failure to implement best practices, either as a result of deficits in health care provider knowledge, attitude (i.e., lack of agreement about the recommendation components), or other contextual factors (i.e., patient preference, practice culture, or environmental influences) (14). Identifying and measuring this gap between evidence and practice is the starting point of knowledge implementation. There are several techniques available that allow us to measure the size and nature of the 'gap'(15), one of which is a needs assessment. A local needs assessment, such as a chart audit, allows us to measure documented clinical care against the quality indicators as set out by HQO.

SD French and colleagues (2012) proposed a four-step systematic method to guide in the development and implementation of knowledge translation interventions; starting with identifying the problem (evidence-to-practice gap), followed by assessing the problem, forming possible solutions, and finally evaluating the selected intervention (16). Utilization of these steps provides health care providers with a strategic approach to a comprehensive needs assessment and identification of evidence-to-practice gaps, with the outcome of locally identified interventions and meaningful outcome measurement. In this paper, 
we describe the process and results of a chart review undertaken to provide further information on identifying the problem (i.e., who needs to do what, differently?) and to gain information on barriers and enablers of early mobility after surgical repair of a hip fracture.

In 2011, an interprofessional team of health care providers at a large urban tertiary centre in Toronto, Ontario implemented the Bone \& Joint National Model for Hip Fracture Care \& Toolkit (2011) on one postoperative unit. Implementation strategies included the introduction of hip fracture specific nursing care pathways, pre-printed order sets for prescribers, and interprofessional staff education sessions. Front line health care providers assisted in the design of the care pathways, integrating recommendations as care prompts. For example, the first post-operative day of the pathway included delirium prevention tactics, prompts for catheter removal and suggestions for early mobility activities. Pre-printed order sets included checklists for weight-bearing as tolerated, pain medication options, and pre-selected options for physiotherapy and occupational therapy referrals. The recent publication of the 2017 HQO Standards and a change in the local knowledge implementation tools present an opportune time to assess practice and identify the presence of evidence-to-practice gaps. We aim to assess the organizations' current practice for initiation of early mobility activities in those patients with fragility hip fracture.

\section{Methods}

\section{Research Setting}

The chart review took place in a large tertiary care centre located in Toronto, Ontario. The hospital admits approximately 200 older adults with hip fracture per year (17). As per HQO recommendations (2), surgical repair of a fragility hip fracture occurs within 48 hours of patient admission. Postoperatively, the majority of these patients are admitted to a shared orthopedic / neurology post-surgical unit. This post-surgical unit has 38 beds, with two full-time physiotherapists, one occupational therapist, and one assistant shared between the therapists. Physiotherapy has limited availability on the weekends; one physiotherapist is responsible for covering five separate postoperative units. There is a four to one patient to nurse ratio during daytime hours.

\section{Data Extraction Instrument}

At this time, there is no standardized data extraction instrument identified by the $2017 \mathrm{HQO}$ Quality Standard for Hip Fractures. A review of the literature identifies several variables that have the potential to impact early mobility after a fragility hip fracture. These variables include age (18-22), sex (20, 23), type of fracture $(18,22)$, a low baseline functional status $(21,22,24,25)$, residence prior to admission , comorbidities $(18,26)$, cognitive impairment $(27,28)$, time to surgery and anesthetic approach $(29)$, pain management (28-31), and medical interventions or environmental barriers $(29,31)$. The data extraction form was designed to include these variables. A pilot audit with a small sample was undertaken to confirm that all the data extraction fields were available within in the hospital chart. We obtained approval from both the hospital Research Ethics Board and Queen's University Health Research Ethics Board to conduct the pilot. The hospital health records department provided a list of patients coded as a 
hip fracture admission from April 1, 2016 to March 31, 2017, and from this list, every tenth chart was selected to a total of ten charts. The chart data extracted for the pilot was not analyzed. This data was used simply to modify the data extraction form to allow for systematic collection of information. Fear of falling has been identified as a variable that may impact early mobility (32); however, this variable was not included in the final data extraction form as it was not observed within any pilot audit documentation. The pilot list of patients was also utilized for the full chart review.

\section{Measurement sources}

For this study, cognitive status was defined by notation of a dementia or delirium diagnosis within the physician note at time of admission. Interprofessional notes were also examined to provide details about cognitive status both prior to surgery and in the post-operative period.

Within the institution, an assessment measure of a patient's pre-fracture functional status is not utilized. In the literature, a frequently referenced reporting measurement representing one's pre-fracture functional status is the New Mobility Score (NMS). The NMS was originally developed to predict mortality after a fragility hip fracture in the older adult population. The NMS is a composite score of a patient's ability to perform indoor walking, outdoor walking, and shopping (33). Direct patient reporting of their preinjury ability provides a score between 0 and $9(0=$ not at all, $1=$ with help from another person, $2=$ with gait aid, and $3=$ no difficulty and no gait aid) for each function. The total score ranges from 0 (no walking ability at all) to 9 (fully independent). The NMS is a reliable and valid tool and has been demonstrated to be an independent predictor of in-hospital outcomes (22), with a lower score (less than five) being a significant predictor of not regaining basic mobility at four months $(21,25,34)$. During data extraction, we aimed for accuracy in score calculation by obtaining NMS data from several sources - social work notes, occupational therapy and physiotherapy notes, and the emergency department record. For this study, we dichotomized the NMS groups - low and high. An NMS of 0-5 was categorized as low, and those scoring over six was categorized as high, or good functional independency.

The Charlson Comorbidity Index (CCl) was used to characterize patient comorbidity. The CCl consists of 19 selected conditions that are weighted, producing a score from 0-33 (35). Statistically significant associations have been demonstrated between a $\mathrm{CCl}$ score greater than or equal to three, and the occurrence of complications after hip fracture surgery (36). Surgical records provided demographic information, anesthetic type, surgical information, and early complications (e.g., intraoperative blood transfusions). Medical orders were reviewed to assess utilization of the pre-printed order sets.

Analysis

Patient characteristics were described using means and standard deviations. Consistent with the 2017 HQO recommendations, achievement of mobility activities (i.e. physiotherapy assessment on the first day after surgery; sit on edge of bed; sit in a chair; mobilize in the room; and mobilize out of the room) was tracked for each patient. In addition to a patient's pre-fracture functional status, the presence of cognitive impairment has been found to be a potential factor affecting a patient's participation in mobility activities 
during their hospital stay (25). Participants were grouped by their pre-fracture functional mobility and cognitive status, and in-group comparisons of achievement of the HQO mobility activities was conducted using Chi Square analysis.

\section{Results}

During the 12 month study period, a total of 145 older adult patients 65 years of age and over were admitted with a diagnosis of hip fracture. We excluded those patients who had a traumatic fracture $(\mathrm{n}=$ 3 ) or did not require surgical repair $(n=11)$, those admitted over 48 hours from time of fracture $(n=2)$, and those with unable to walk independently prior to their fracture (NMS less than $2, n=11$ ). As this study aims to examine practices in one specific unit, any patients transferred to other units during hospitalization were excluded $(n=40)$. We excluded one patient who died during surgery. The final analysis included of a total of 77 patients admitted to the target unit (Fig. 1).

Of the 77 patients, 32 (42\%) were over the age of 90 years, 33 (43\%) had dementia listed as a comorbidity on admission and were allocated to the cognitively impaired group, 37 (48\%) of patients had a low pre-fracture functional mobility level (Table 1). A total of $43(68 \%)$ of patients were living at home in the community prior to admission. Of the $12(16 \%)$ living in a retirement residence, eight had a cognitive impairment, and all $12(16 \%)$ admitted from long term care had a cognitive impairment.

The mean time to surgery in the cohort was 33 hours (SD:17.16), with a range from 3 to 97 hours, 7 (9\%) patients had their surgery beyond the recommended 48 hours after admission. The average time to surgery was not significantly different between those with no cognitive impairment (35 hours, with four patients after 48 hours) and those with a cognitive impairment (31 hours, with three patients after 48 hours) $(p=.688)$. Of the patient group that developed post-operative cognitive changes, eight patients had no history of cognitive impairment documented on admission (Table 2). As patients were discharged, the patient sample size decreased, by post operative day five $38(49 \%)$ had been discharged, with an end sample size of 39 (Table 2).

Mobility activities

On the first post-operative day, 71 (92\%) of the cohort patients were assessed by physiotherapy. The rate of physiotherapy assessments declined over the following post operative days. $100 \%$ of the low prefracture mobility patients with no cognitive impairment were assessed by physiotherapy the first day, compared to $82 \%$ of those with a high pre-fracture mobility and cognitive impairment (Table 2).

Conversely, on the fifth day, all five patients in the high pre-fracture function with a cognitive impairment were assessed by physiotherapy, compared to only $36 \%$ of those with a low pre-fracture function and cognitive impairment (Table 2). There was a significant difference observed on the second post operative day in the low pre-fracture mobility groups; physiotherapists were significantly more likely to assess those with a cognitive impairment than those with no cognitive impairment $(p=.031)$ (Table 2). 
Achieving mobility to chair was documented consistently by both physiotherapy and nursing staff. Only $39(51 \%)$ of patients were transferred to chair on the first day, with subsequent post-operative days demonstrating an increasing number of the patients being up to chair (Fig. 1). Interestingly, the lowest percentage of mobility to chair was represented by those with a high pre-fracture function with no cognitive impairment on the first day after surgery (45\%), yet by the fourth day after surgery, this was the group with the highest percentage of mobility to chair (89\%) (Table 2). Those with low pre-fracture function and a cognitive impairment demonstrated highest rate of mobility to chair on the third day ( $84 \%$ ) (Table 2).

On the first day, 35 (45\%) of patients walked within the room (less than 5 metres). Patients with a low prefracture function had a higher incidence of achieving this activity on the first day (64\%), yet this group's documented participation in this activity markedly declined in the following days to the lowest on the fifth post operative day (21\%) (Fig. 2). Those with a low pre-fracture function and no cognitive impairment were significantly more likely to achieve this activity than their cognitively impaired peers $(p=.035)$ (Table 2). The highest percentage of walking within the room was represented by those with a high prefracture function with no cognitive impairment on the second day after surgery (76\%) (Table 2). When comparing groups across all activities, there were no other significant findings.

Overall, only 5 (6\%) of patients were able to ambulate over 5 meters on the first post-operative day. By postoperative day five, across all groups, only two patients walked over 50 metres (one patient walked 200 metres). Only those with a high pre-fracture function achieved this milestone within the first three days post surgery. By the fourth day, at least one patient from all groups walked out of room. The highest percentage of walking out of room was represented by those with a high pre-fracture function with no cognitive impairment on the second day (31\%) (Table 2).

Pain and medical complications

Twelve (16\%) of patients had a documented report of pain, and ten (13\%) were documented to have a medical issue leading to a deferral of physiotherapy (e.g, x-ray, telemetry, low blood pressure). The Classification of Surgical Complications was utilized to describe postoperative complications of the cohort. Within the cohort, $44(57 \%)$ had no complications. Ten (13\%) patients displayed a Grade 1 complication. The most common complication was receiving a blood transfusion (Grade 2) by $16(21 \%)$ patients. Seven patients (9\%) died during their hospital stay (Grade 5). The mean length of postoperative stay was seven days (range 1-64 days), with 42 (55\%) of the patients discharged by postoperative day five. All sixty-one (79\%) patients admitted from the community were discharged to a rehabilitation facility, and ten $(13 \%)$ were discharged directly to their previous long-term care facility.

\section{Discussion}

Since the institutions' implementation of the Bone \& Joint Canada - Hip Fracture Toolkit recommendations in 2011, the nursing care pathways have been revised to be more broadly applicable a wide range of post-surgical populations, and hip fracture specific prompts for post-operative care were 
removed from the pathway in 2015 . We did not know if the care prompts served as important reminders for the nursing staff, and we did not know the level of early mobility activities undertaken by health care providers. The 2017 publication of the HQO recommendations prompted a needs assessment to identify local evidence-to-practice gaps that may exist in the area of early mobility after surgery. Returning to the French \& Green [16] four-step method, the chart audit information will be utilized to work through, specifically, step one: identifying if an evidence-to-practice gap exists, clinical behaviours that may be a target for change and who performs these behaviours, and when / where are these behaviours performed.

Identifying the evidence-to-practice gap

To identify the evidence-to-practice gap, we will compare the results of the chart review with HQO Standards (2017) (2).

HQO 2017 Standard 8: "Ensure protocols, pathways, medical directives, and standardized order sets are in place to facilitate patients achieving weight-bearing as tolerated within 24 hours following surgery" (p. 23). The institution's adoption of HQO recommendations in 2009 had set out that the surgical teams are to repair the fragility fracture in a method that allows for early total weight-bearing, thereby avoiding the potential for prolonged bedrest post-operatively. Following surgery, prescribers also have the responsibility of ordering several interventions aimed at facilitating early mobility: a) weight-bearing as tolerated, b) physiotherapist assessment immediately after surgery, c) appropriate pain management medication, and d) early removal of medical interventions (i.e., urinary catheter and intravenous fluids). The pre-printed order sets with checklists for weight-bearing as tolerated, pain medication options, and automatic referrals to physiotherapy and occupational therapy were utilized in $99 \%$ of the cases.

HQO 2017 Standard 9: "Following surgery, ensure patients with hip fracture are mobilized at least once daily by a member of the health care staff. Where possible, family members or caregivers should be encouraged to assist with mobilization" (p. 26). Within the Standard, HQO defines mobility as a progression through a continuum of functional activities (i.e., sit at bedside, transfer to chair with assistance, transfer to chair independently, walk with assistance) (2). It is important to note that the definition of expected outcomes is not clearly stated within the Standard, nor does it indicate an outcome for measurement. Thus, it was difficult to ascertain what percentage of patients 'met' the criteria of daily mobility due to this lack of a clear definition. However, our data indicate a range of $50 \%-89 \%$ of patients got up to chair, meaning that $11 \%-50 \%$ are not participating in this activity in the first five days, and thus potentially not meeting HQO criteria. Even though there was a high rate of physiotherapy assessments on the first day (92\%), only $51 \%$ of patients were able to get up to chair. When pre-fracture function is taken into consideration, one would expect that those patients with high pre-fracture function would be better able to participate in these activities, but only $45 \%$ of this group was up to chair on the first day after surgery. There may be several reasons for these low rates of participation - it is unclear if patients are not participating in the activity, or the documentation is not reflective of practice. The physiotherapists document each progressive activity performed, whereas the nursing documentation is more general, primarily reflecting time spent in chair. As physiotherapy assessments decline over time, the chart review 
data changes from identification of specific tasks (i.e., edge of bed) to time in chair. A further study of practices on the unit may be required to assess the actual rates of participation. The differences in documentation style, and lack of consistent language within the documentation makes it difficult to determine patient progression throughout the post-operative days.

One of the HQO recommended progressive activities is walking with assistance, yet no identification of goals for distances are provided. Within our cohort, by postoperative day five, only two patients in the high pre-functional group walked over 50 metres, with one patient walking 200 metres. Similar findings of limited tolerance for walking have been reported during the acute post operative recovery phase $(28,37)$. Literature has suggested that assessment of functional level and utilization of care pathways based on pre-fracture functional status may be feasible (34).

The HQO standards recommend that patients receive daily physiotherapy and occupational therapy, regardless of a patient's cognitive status. Cognitive impairment $(\mathrm{Cl})$ has been identified as one of the most common barriers to early mobilization (38). However, in our chart review, in the first two days after surgery this population has a higher rate of achieving mobility activities than those with no cognitive impairment (Chart 1). Of the $48 \%$ that experienced some level of cognitive impairment, either from admission or new-onset after surgery, $54 \%$ patients were up to chair on the first day after surgery, compared to only $48 \%$ of those with no cognitive impairment. By the second day, those with no cognitive impairment have a higher rate of up to chair. The highest occurrence of up to chair for the cognitively impaired group did not occur until post operative day three (78\%). The rationale for the differences between pre-fracture functional groups and cognitive status are unclear from the chart review, further assessment of local factors which may be influencing these findings is needed.

Clinical behaviours that may be a target for change (who performs the behaviours)

HQO (2017) has outlined daily mobilization should be facilitated by all members of the healthcare team, inclusive of family members. The chart review has confirmed that prescribers are utilizing the pre-printed order sets, not requiring further intervention at this time. There was no documentation within the physiotherapy or nursing notes about the inclusivity of family members in early mobility activities. This finding is interesting in that one wonders the extent to which family members are educated by staff to be involved in mobility activities, and even if they are involved, do the health care provider staff feel that family involvement is a documentable intervention? Family member involvement in mobility activities is an area of interest that would be worth exploring directly with the patients and their family members. It would be worthwhile to assess if a patient's cognitive status impacts the level of involvement from family members. The patient and family member involvement may be an opportune target for behaviour change.

HQO (2017) indicates any health care provider involvement, including nursing, should be utilized to facilitate early mobility. In our chart audit, the mobility activity documented most by nursing staff was time spent in chair. The nursing documentation tool also has blank areas for 'sitting at edge of bed', 'sit to stand', 'ambulation', but this information was rarely completed. It is unclear whether this incomplete 
nursing documentation reflects a practice gap or inconsistent/missing documentation. Studies have suggested that the most frequent nurse initiated mobility activities in the older adult population are standing and transferring (39). It would be of interest to ask nurses if they consider the associated activities (i.e., sit at edge of bed) when they are documenting the patient time in chair. There was very little evidence of nursing proceeding to walk with patients. There is evidence to suggest that nurses may experience barriers with early mobility in this older adult population. Studies have identified that fear of the patient falling, lack of assistive devices, and lack of staff influenced nurses' decisions about ambulating older patients (31). Doherty-King and Bowers (40) also found that nurses perceive risks related to injury to the patient (fall) or self (back) if they engaged older patients in walking. Further conversations with the nursing team about their barriers and facilitators to mobility activities with patients after hip fracture is warranted.

Literature has identified barriers to rehabilitation in patients with $\mathrm{Cl}$ symptoms, including a limited ability to initiate activities; a demonstrated lack of insight/judgment, loss of purposeful movements, loss of recognition; and memory problems or language impairments (41). It is not clear from the chart review documentation if health care providers limit their time with patients related to facilitating early mobility activities in this patient population. Not all health care providers providing direct patient care have equal knowledge about effective strategies for rehabilitation in those with $\mathrm{Cl}$, resulting in diminished health care processes (41). Our chart review data confirms that there is an apparent disparity of physiotherapy assessments with those who are documented to have a cognitive impairment and low pre-fracture functional ability. This area would be one of interest to explore with health care providers.

Previous studies have identified medical interventional barriers such as intravenous lines, oxygen tubing, and urinary catheters (31). In our chart review, these barriers did exist; however, there was no documentation of these items listed as a cause of being unable to mobilize a patient. Additionally, we could find no mention of solutions or alternatives to address these tangible barriers (e.g., intravenous disconnected to allow for mobility). Based on this chart review alone, it is unclear if these medical interventions are a perceived barrier by health care providers or patients.

When and where are these behaviours performed

Although not specifically outlined by the HQO standard, one could picture the early mobility progression as a patient being assisted to sit at the edge of bed, transfer to a chair for meals, participate in physiotherapy, and continue with exercises as tolerated with the help of health care staff or family members. Performance of daily personal care and activities of daily living would be an opportune time for patients to engage in mobility activities - for example, having a patient ambulate to the bathroom for daily hygiene activities. Early mobility activities are the responsibility of the entire health care team, inclusive of a preliminary assessment by the physiotherapy team, involvement of occupational therapy, and finally incorporating education about early mobility for assistance by family members.

As stated, current documentation is fragmented across health care providers, making it difficult to track progress in mobility activities throughout a patient's stay. Additionally, there was no information reflective 
of the patient and/or family perspective about early mobility activities, this may be a potential area for future change.

\section{Limitations}

The chart review was undertaken as a needs assessment to analyze presence of an evidence-to-practice gap. The chart review method is limited by several factors. As a planned strategy to minimize errors of omission, only one person (LH) conducted the actual review. LH was involved in the initial implementation intervention, is aware of changes to documentation over the years, and has an extensive knowledge of location of where information was likely to be documented within the live electronic and scanned chart. There was a large percentage of undocumented data. The strength of the audit is only as strong as the data recorded in the chart. If the data was not recorded, it was assumed that the task was not done. This potentially underestimates what activities were facilitated by the health care providers.

Evidence-to-practice gaps often occur secondary to systems issues and not solely on provider performance (15). The chart audit is limited in its ability to assess the systems issues, which may have an influence on the health care provider behaviour. Previous work identified that nursing team involves physiotherapy to ensure safe mobilization of patients (31). It would be interesting to explore further the concept of the relationship between all health care providers to better understand how the team works together to facilitate early mobility activities.

\section{Conclusions}

Our chart review has reported similar findings to others in the literature, namely, that older adults experiencing a fragility hip fracture are slow to mobilize after surgery. We have identified the areas where recommendations have been implemented successfully, and potential areas of evidence-to-practice gaps. The chart review has uncovered a paucity of contextual information which may influence health care providers' behaviours related to early mobility. Considering the lack of information in these areas, we recognize that further work is required to explore factors which may be having an impact on the health care provider's ability to engage the patients in early mobility activities. There are several additional strategies, such as direct observation, and health care provider interviews, which may be better suited to examine factors that influence the use of evidence in day to day practice.

A prospective exploratory embedded case study (Phase 2) is planned to explore the contextual factors influencing activities undertaken by the health care providers and patients. Many decisions that health care providers make at the point of care are context-dependent (16). It is planned to continue to follow the French four-step method (16) to identify the pathways of change, and possible barriers and enablers to behaviour change within our organization.

\section{Abbreviations}

NICE National Institute of Health and Care Evidence 
HQO Health Quality Ontario

NMS New Mobility Score

$\mathrm{CCl} \quad$ Charleston Co-morbidity Index

LTC Long term care

IM nail Intramedullary nail

GA General Anesthetic

$\mathrm{Cl} \quad$ Cognitive impairment

\section{Declarations}

Ethics approval and consent to participate: Ethics approval was obtained from the institute in which the research took place, as well as through Queen's University.

Consent for publication: As it was a retrospective chart review, consent forms were not required.

Availability of data and material: The datasets during and/or analysed during the current study available from the corresponding author on reasonable request.

Competing interests: The authors declare that they have no competing interests

Funding: The project was funded in part by the Sunnybrook Practice Based Research Institute.

Authors' contributions: LH collected, analyzed and interpreted patient data, and was lead in writing the manuscript. All authors read and approved the final manuscript.

Acknowledgements: Not applicable

\section{References}

1. Osteoporosis Canada. Osteoporosis Canada; facts and statistics http://www.osteoporosis.ca/osteoporosis-and-you/osteoporosis-facts-and-statistics/2015 [

2. Health Quality Ontario. Quality Standards. Hip fracture care for people with fragility fractures. Toronto2017.

3. Eastwood EA, Magaziner J, Wang J, Silberzweig SB, Hannan EL, Strauss E, et al. Patients with hip fracture: subgroups and their outcomes. J Am Geriatr Soc. 2002;50(7):1240-9. 
4. Penrod JD, Litke A, Hawkes WG, Magaziner J, Koval KJ, Doucette JT, et al. Heterogeneity in Hip Fracture Patients: Age, Functional Status, and Comorbidity. Journal of American Geriatrics Society. 2007;55:407-13.

5. Auais M, Morin S, Nadeau L, Finch L, Mayo N. Changes in frailty-related characteristics of the hip fracture population and their implications for healthcare services: evidence from Quebec, Canada. Osteoporos International. 2013;24(10):2713-24.

6. Magaziner J, Simonsick EM, Kashner TM, Hebel JR, Kenzora JE. Predictors of functional recovery one year following hospital discharge for hip fracture: a prospective study. J Gerontol. 1990;45.

7. Ruggiero C, Bonamassa L, Pelini L, Prioletta I, Cianferotti L, Metozzi A, et al. Early post-surgical cognitive dysfunction is a risk factor for mortality among hip fracture hospitalized older persons. Osteoporosis International. 2017;28(2):667-75.

8. Krenk L, Rasmussen LS. Postoperative delirium and postoperative cognitive dysfunction in the elderly - what are the differences? Minerva Anestesiol. 2011;77(7):742-9.

9. Seitz DP, Gill SS, Austin PC, Bell CM, Anderson GM, Gruneir A, et al. Rehabilitation of Older Adults with Dementia After Hip Fracture. J Am Geriatr Soc. 2016;64:47-54.

10. National Insitute of Health and Care Evidence (NICE). Clinical guideline: Hip fracture management. National Institute for Health and Care Excellence (NICE); 2017.

11. Oldmeadow LB, Edwards ER, Kimmel LA, Kipen E, Robertson VJ, Bailey MJ. No rest for the wounded: early ambulation after hip surgery accelerates recovery. ANZ J Surg. 2006;76(7):607-11.

12. McGlasson R, MacDoald V, Lo N, Spafford D, McMullan JL, Beaupre L, et al. National Hip Fracture Toolkit. Bone and Joint Canada; 2011.

13. S.I.G.N. Management of Hip Fracture in Older People: A National Clinical Guideline. Scottish Intercollegiate Guidelines Network (SIGN); 2009.

14. Legare F, Zhang P. Barriers and Facilitators. Strategies for identification and measurement. In: Straus S, Tetroe J, Graham ID, editors. Knowledge Translation in Health Care: Moving from Evidence to Practice. 2nd ed: John Wiley \& Sons; 2013.

15. Kitson AL, Straus SE. Identifying knowledge to action gaps. In: Straus SE, Tetroe J, Graham ID, editors. Knowledge translation in health care; moving evidence to practice. 2nd ed: John Wiley \& Sons, Ltd; 2013.

16. French SD, Green SE, O'Connor DA, McKenzie JE, Francis JJ, Michie S, et al. Developing theoryinformed behaviour change interventions to implement evidence into practice: a systematic approach using the Theoretical Domains Framework. Implementation Science. 2012;7(1):38.

17. GTA Rehab Network. [Available from: http://www.gtarehabnetwork.ca/.

18. Koot V, Peeters P, de Jong J, Clevers G, van der Werken C. Functional Results after Treatment of Hip Fracture: a Multicentre, Prospective Study in 215 Patients. Eur J Surg. 2000;166:480-5.

19. Siu A, Penrod JD, Boockvar K, Koval K, Strauss E, Morrison S. Early Ambulation After Hip Fracture Effects on Function and Mortality. Arch Intern Med. 2006;166:766-71. 
20. Solarino G, Vicenti G, Picca G, Rifino F, Carrozzo M, Moretti B. A review of gender diff erences in hip fracture anatomy, morbidity, mortality and function. Ital J Gender-Specific Med. 2016;2(2):55-9.

21. Hulsbaek S, Larsen RF, Troelsen A. Predictors of not regaining basic mobility after hip fracture surgery: A systematic review and meta-analysis. Disabil Rehabil. 2015;37(19):1739-44.

22. Kristensen MT, Foss NB, Ekdahl C, Kehlet $H$. Prefracture functional level evaluated by the New Mobility Score predicts in-hospital outcome after hip fracture surgery. Acta Orthop. 2010;81(3):296302.

23. Wehren L, Hawkes W, Orwig D, Hebel JR, Zimmerman SI, Magaziner J. Gender Differences in Mortality After Hip Fracture: The Role of Infection. Journal of Bone and Mineral Research. 2003;18(12).

24. Foss NB, Kristensen MT, Kehlet H. Prediction of postoperative morbidity, mortality and rehabilitation in hip fracture patients the cumulated ambulation score. Clinical Rehabilitation. 2006;20(701-708).

25. McGilton KS, Chu CH, Naglie G, Wyk PM, Stewart S, Davis AM. Factors Influencing Outcomes of Older Adults After UndergoingRehabilitation for Hip Fracture. Journal of the American Geriatrics Society. 2016;64(8):1601-9.

26. Carpintero P, Caeiro J, Carpintero R, Morales A, Silva S, Mesa M. Complications of hip fractures: A review. World J Orthop. 2014;5(4):402-11.

27. Morandi A, Davis D, Fick DM, Turco R, Boustani M, Lucchi E, et al. Delirium Superimposed on Dementia Strongly Predicts Worse Outcomes in Older Rehabilitation Inpatients. Journal of the American Medical Association. 2014;15(5):349-54.

28. Munter KH, Clemmesen CG, Foss NB, Palm H, Kristensen MT. Fatigue and pain limit independent mobility and physiotherapy after hip fracture surgery. Disabil Rehabil. 2017:1-9.

29. Siu AL, Boockvar KS, Penrod JD, Morrison RS, Halm EA, Litke A, et al. Effect of inpatient quality of care on functional outcomes in patients with hip fracture. Med Care. 2006;44.

30. Sims-Gould J, Stott-Eveneshen S, Fleig L, McAllister M, Ashe MC. Patient Perspectives on Engagement in Recovery after Hip Fracture: A Qualitative Study. J Aging Res. 2017;2017:2171865.

31. Brown CJ, Williams BR, Woodby LL, Davis LL, Allman RM. Barriers to mobility during hospitalization from the perspectives of older patients and their nurses and physicians. J Hosp Med. 2007;2(5):30513.

32. Oude Voshaar RC, Banerjee S, Horan M, Baldwin R, Pendleton N, Proctor R, et al. Fear of falling more important than pain and depression for functional recovery after surgery for hip fracture in older people. Psychological Medicine. 2006;36(11):1635-45.

33. Parker MJ, Palmer CR. A new mobility score for predicting mortality after hip fracture. J Bone Joint Surg Br. 1993;75(5):797-8.

34. Pedersen TJ, Lauritsen JM. Routine functional assessment for hip fracture patients. Are there sufficient predictive properties for subgroup identification in treatment and rehabilitation? Acta Orthop. 2016;87(4). 
35. de Groot V, Beckerman H, Lankhorst GJ, Bouter LM. How to measure comorbidity: a critical review of available methods. J Clin Epidemiol. 2003;56:221-9.

36. Flikweert ER, Wendt KW, Diercks RL, Izaks GJ, Landsheer D, Stevens M, et al. Complications after hip fracture surgery: are they preventable? European Journal of Trauma and Emergency Surgery. 2018;44(4):573-80.

37. Taraldsen K, Sletvold O, Thingstad P, Saltvedt I, Granat MH, Lydersen S, et al. Physical behavior and function early after hip fracture surgery in patients receiving comprehensive geriatric care or orthopedic care--a randomized controlled trial. J Gerontol A Biol Sci Med Sci. 2014;69(3):338-45.

38. Resnick B, Beaupre L, McGilton K, Galik E, Liu W, Neuman MD, et al. Rehabilitation interventions for older individuals with cognitive impairment post-hip fracture: a systematic review. Journal of PostAcute and Long-Term Care Medicine. 2016;17:200-5.

39. Doherty-King B, Yoon JY, Pecanac K, Brown R, Mahoney J. Frequency and duration of nursing care related to older patient mobility. J Nurs Scholarsh. 2014;46(1):20-7.

40. Doherty-King B, Bowers B. Attributing the responsibility for ambulating patients: a qualitative study. International Journal of Nursing Studies. 2013;50:1240-6.

41. McGilton K, Wells J, Davis A, Rochon E, Calabrese S, Teare G, et al. Rehabilitating Patients With Dementia Who Have Had a Hip Fracture Part Il: Cognitive Symptoms That Influence Care. Top Geriatr Rehabil. 2007;23(2):174-82.

\section{Tables}

Due to technical limitations, the tables are only available as a download in the supplemental files section.

\section{Figures}




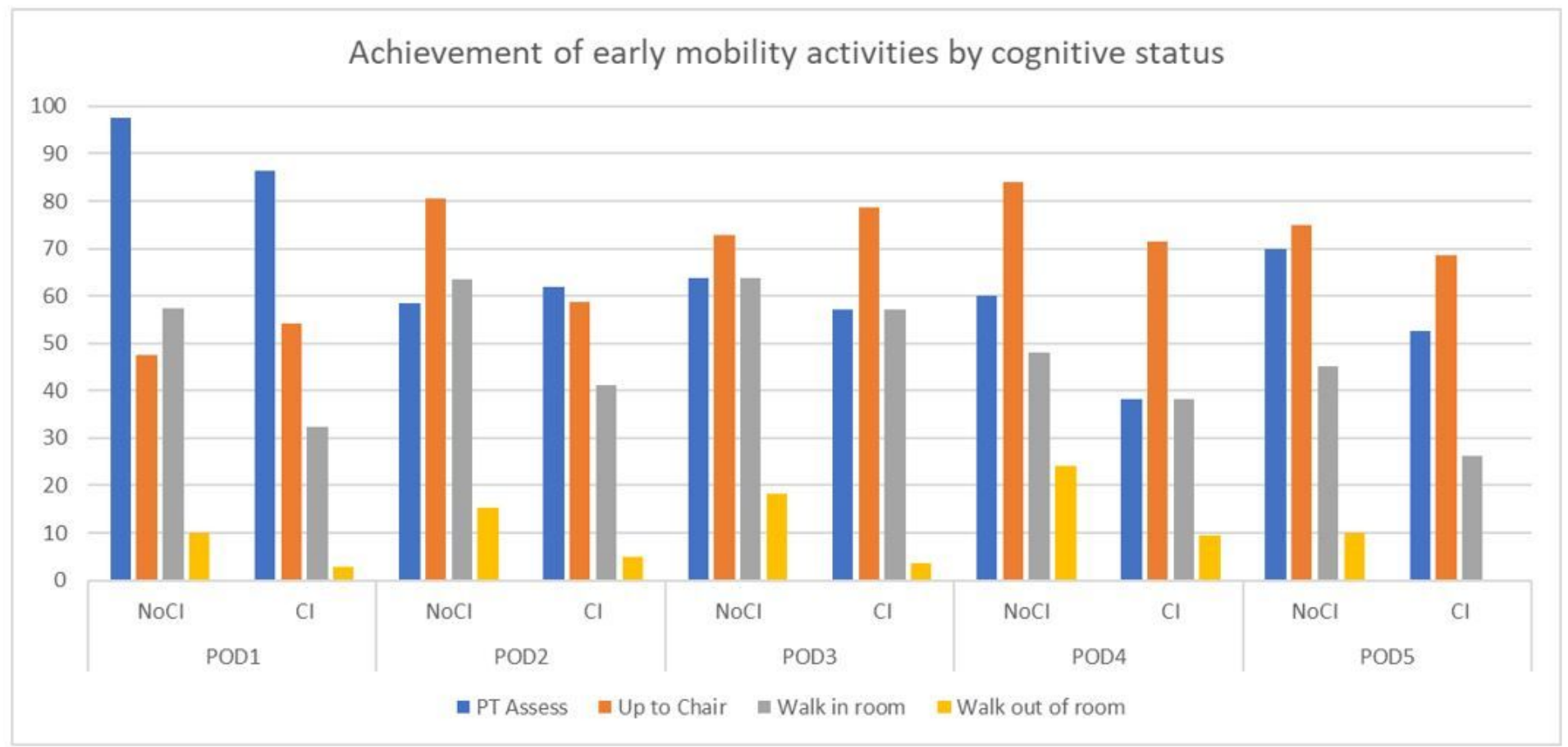

Figure 2

Achievement of early mobility activities by cognitive status

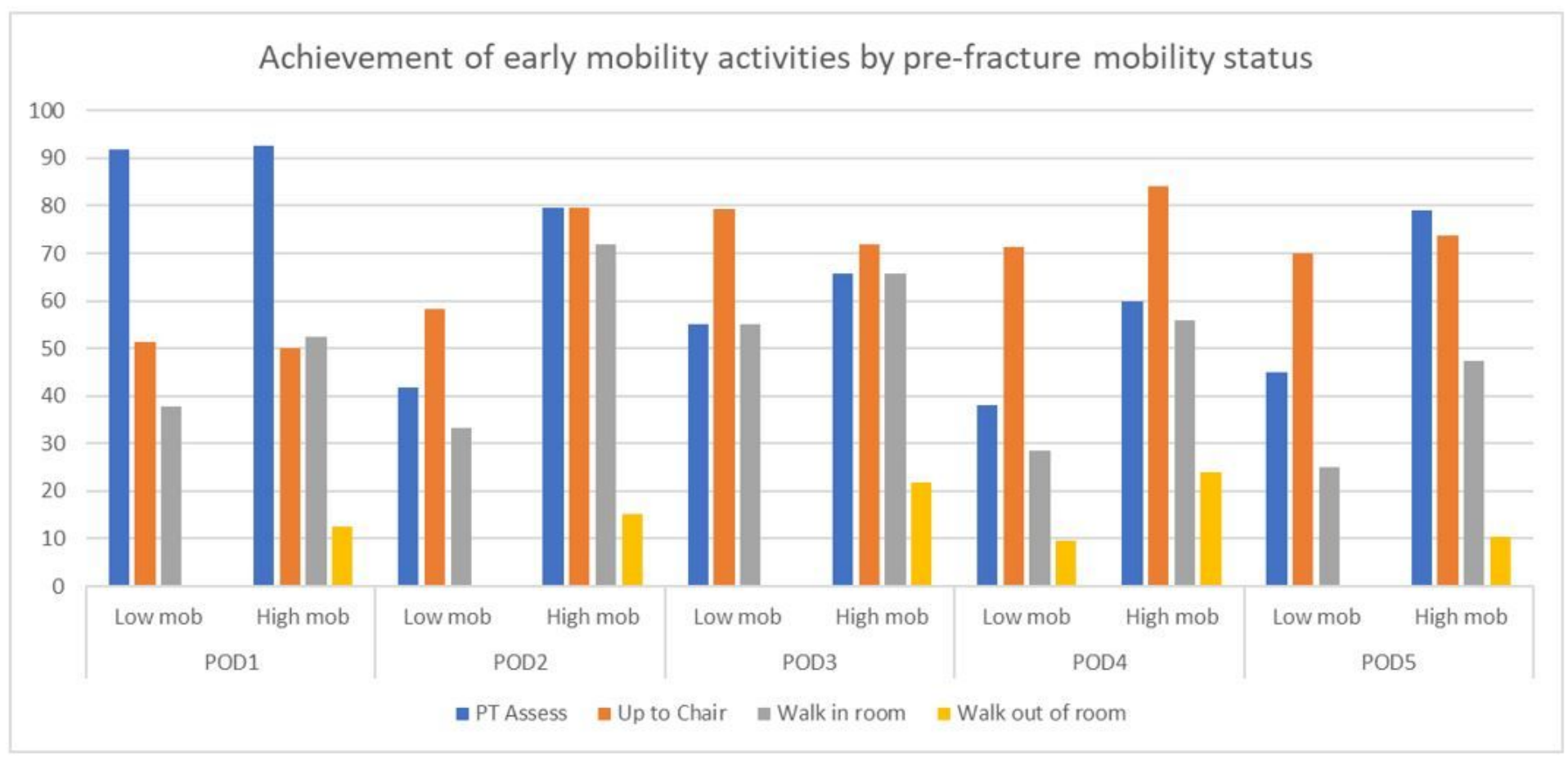

\section{Figure 3}

Achievement of early mobility activities by pre-fracture mobility status

\section{Supplementary Files}


This is a list of supplementary files associated with this preprint. Click to download.

- CONSORTdiagram.docx

- STROBEchecklistcohorthaslam.docx

- Table2submit.xlsx

- Table2submit.xlsx

- Table1chartsubmit.xlsx

- Table1chartsubmit.xlsx

- AppendixAFrenchmethod.docx

- AppendixAFrenchmethod.docx

- STROBEchecklistcohorthaslam.docx

- CONSORTdiagram.docx 